\title{
14. Sur le rôle du nom commun dans le choix entre les appositions des trois types " le président Obama ", " Obama, le président " et "le président, Obama " en français et en suédois
}

Karin Lindqvist

Stockholms universitet

\section{Introduction}

L'extrait suivant du Monde illustre l'importance de présenter et d'identifier les personnages, et d'autres concepts, figurant dans un texte :

(I) Une belle promenade dans la diversité musicale d'Istanbul

Fatih Akin est allemand, né à Hambourg il y a trente-deux ans. Dans ses films, le cinéaste fait souvent l'aller-retour entre son pays natal et la Turquie. Crossing the Bridge [...] n'est pas le récit d'un voyage (contrairement à Im Juli ou Head On), mais celui du séjour d'Alexander Hacke à Istanbul. [...]

Au fil des déplacements à travers Istanbul, les facettes s'agrègent les unes aux autres : la jeunesse éclatante d'Aynur répond à la séduction légèrement frelatée d'Orhan Gencebay, par ailleurs authentique virtuose du saz. [...] Muyezzen Senar renvoie aux temps ottomans, fait deviner un continuum raffiné qui irait d'Istanbul au Caire. [...] (Le Monde, $\mathrm{I} 3 / 07 / 2005$, nos italiques)

Certes, l'extrait ne pose pas de problèmes en ce qui concerne la lisibilité, mais on a l'impression qu'il manque quelque chose. On ne sait pas quelle est la relation entre Fatih Akin et la Turquie. On ne peut que supposer qu'Im Juli et Head On sont des films. Le lecteur " moyen » français ne peut pas être censé connaître Alexander Hacke, ni Aynur, ni Orhan Gencebay, ni Muyezzen Senar. On comprend que le saz doit être un instrument, mais on ne sait peut-être pas de quel genre.

Comment citer ce chapitre:

Lindqvist, Karin, Sur le rôle du nom commun dans le choix entre les appositions des trois types «le président Obama ", "Obama, le président » et "le président, Obama " en français et en suédois. In: Engwall, Gunnel \& Fant, Lars (eds.) Festival Romanistica. Contribuciones lingüísticas - Contributions linguistiques - Contributi linguistici Contribuições linguísticas. Stockholm Studies in Romance Languages. Stockholm: Stockholm University Press. 201 5, pp. 273-290. DOI: http://dx.doi.org/IO.I6993/bac.n. License: CC-BY 
De l'extrait original du Monde, nous avons enlevé les noms communs $(\mathrm{Nc})$ positionnés à côté des noms propres (Npr). Dans l'original, le pays, les films, les personnes et l'instrument sont présentés ainsi : " celui de ses parents, la Turquie ", "ses fictions, Im Juli ou Head On ", "Alexander Hacke [à Istanbul], bassiste au sein du groupe Einstürzende Neubauten, un des fondateurs du rock industriel », " la chanteuse kurde Aynur ", "Orhan Gencebay, étoile des années I970 ", " saz (luth à long manche) ", " la diva octogénaire Muyezzen Senar ». Ces combinaisons de Nc et de Npr sont des exemples d'appositions, le sujet de cette étude, et l'exemple montre l'importance des appositions dans la prose journalistique, où il est, comme le dit Meyer (1992: 6) " communicatively necessary to name individuals and to provide information about them ${ }^{\mathrm{I}}$. Il existe trois types d'appositions, illustrés ici :

(2) apposition liée : la procureure de la République de Lille MarieMadeleine Alliot (NcNpr)

(3) apposition détachée : le guitariste du groupe de rock The Kills, Jamie Hince ( $\mathrm{Nc}[$ détachement $] \mathrm{Npr})$

(4) apposition détachée : Benjamin Netanyahu, le Premier ministre israélien $(\mathrm{Npr}[$ détachement $] \mathrm{Nc})$

Le but du présent article est d'examiner et de comparer l'emploi de ces constructions en français et en suédois écrits. Plus précisément, nous examinerons le rôle que joue l'expansion du Nc : son interprétation sémantique / pragmatique et sa forme, dans le choix entre les trois types d'appositions.

Comme point de départ, citons d'abord ce que dit I. Korzen (2006 : I08) à propos du type d'apposition montré dans (2). Cette apposition constitue, d'après lui, " a particularly cohesive and indivisible unit ». Même avec une expansion dans le Nc, cette forte cohésion resterait inchangée, comme dans son exemple (il s'agit, comme on peut le voir, de la langue italienne) : "il presidente della Repubblica Sandro Pertini " (2006 : I Iо). Citons ensuite cet exemple (anglais) d'AcuñaFariña (2009 : 472) : "the stubborn Jaguar boss Tom Walkenshaw ». A propos du Nc the stubborn Jaguar boss, Acuña-Fariña écrit que cette unité :

[has] enough elaboration to activate a referent by itself. That is, it starts sounding like a referential NP. In that case, the prosodic dissociation of the newly-created NP from the proper noun becomes more necessary given the syntactic weight UI has. 
On peut, à partir de ces citations (pourvu qu'on accepte que les mêmes descriptions peuvent s'appliquer au français et au suédois) tirer la conclusion que le lien entre le Nc et le Npr dans la construction (2) est forte, mais qu'il peut être rompu dans le cas où le Nc est suffisamment élaboré. Dans ce cas, le Nc et le Npr sont « dissociés ». Dans la langue écrite, cette dissociation prosodique peut vraisemblablement se traduire par un détachement graphique, qui résulte en le type d'apposition montrée dans (3). Le type dans (4) contient aussi un Nc et un Npr, et constitue donc un autre choix possible. Nous examinerons dans quelle mesure l'interprétation sémantico-pragmatique du Nc régit le choix entre les constructions. Notre hypothèse est que l'interprétation du Nc comme référentiel ou non dépend aussi de la proximité entre la tête du $\mathrm{Nc}$ et du Npr : si ces deux noms (N) sont contigus, on tend à les interpréter comme un seul groupe nominal $(\mathrm{GN})$ référentiel, et dans ce cas, il est moins probable que les deux $\mathrm{N}$ soient dissociés graphiquement.

L'étude traitera donc de la langue écrite journalistique, française et suédoise, et on appliquera une perspective contrastive au problème, à cause de la comparaison entre le français et le suédois en soi, mais aussi parce qu'en comparant ces deux langues, il ressort clairement combien la forme de l'expansion affecte le choix de construction.

\section{Présentation théorique et hypothèse}

\subsection{Terminologie et description syntaxique}

Acuña-Fariña (2009: 458) soutient que l'apposition liée et l'apposition détachée "are in fact two radically different things ". C'est une conception qu'il partage avec Forsgren (I99I), H. Korzen (2006) et I. Korzen (2006). Acuña-Fariña décrit l'apposition détachée comme une insertion pragmatique qui ne participe pas à la formation syntaxique de la phrase ${ }^{2}$, alors que les appositions liées sont décrites comme "instances of more or less ordinary NP structure » (Acuña-Fariña, 2009 : 458). Malgré les divergences, on emploie souvent le terme " apposition " pour les deux variantes, celle sans détachement et celles avec détachement. Les deux types illustrés dans (3) et (4) sont appelés « apposition » par ex. par Forsgren (2000) pour le français, et par Teleman et al. (I999) pour le suédois (et d'ailleurs aussi pour l'anglais par Acuña-Fariña (2009)3). Le type dans (2) est appelé " apposition » par ex. par Rioul (I983)4 et par Teleman et al. (1999) (et pour l'anglais par Keizer, 2005).

Pour ce qui est de l'analyse syntaxique des deux types de construction, c'est là un problème beaucoup discuté, dont les avis des chercheurs 
diffèrent souvent. Forsgren (2000) considère par exemple l'apposition à détachement comme une fonction syntaxique, à côté de l'épithète et de l'attribut, alors qu'Eriksson (I993) la décrit comme une relation syntaxique, à côté de la coordination et de la subordination. Pour ce qui est du lien entre les deux GN dans la construction, c'est là une autre question débattue. Selon I. Korzen (2006), le segment détaché ne peut pas être considéré comme appartenant au GN dans la phrase enchâssante. Il montre que l'apposition détachée peut être analysée comme un constituant syntaxique n'appartenant ni aux constituants primaires (le verbe, le sujet, les objets, etc.) ni aux constituants secondaires (les modificateurs et les déterminants). L'apposition détachée constitue un groupe à part, selon cette analyse. Hannay et Keizer (2005) font une analyse similaire, mais appliquent une perspective plutôt discursive au problème. Les appositions détachées sont décrites comme des unités discursives indépendantes ne faisant pas partie de la phrase enchâssante : " holophrases, being non-sentential in form, but making a full and complete contribution to the discourse " (Hannay \& Keizer, 2005 : I65). Quant à la construction sans détachement, elle a aussi été beaucoup discutée, surtout concernant la question de savoir si on peut considérer un des deux GN comme une tête par rapport à l'autre, et, dans ce cas, lequel des deux. Il est en effet possible de trouver des chercheurs qui analysent soit le premier des deux GN comme une tête (par ex. Forsgren 200I), soit le deuxième (par ex. Halmøy 200I), soit les deux à la fois (cf. Jonasson I994, qui soutient que "[1]e Npr et le Nc fonctionne [sic] tous les deux comme têtes d'un GN complexe, aucun d'eux n'étant à voir comme le modifieur ou le complément de l'autre »).

Après ce petit exposé, il faut constater qu'une description complète de ces problèmes très complexes dépasserait de loin le cadre de l'étude, mais aussi que le but du travail est d'examiner les deux types de construction dans leur emploi concret. Par conséquent, nous ne développerons pas davantage la présentation théorique.

\subsection{Le choix entre les constructions : hypothèse}

Comme il vient d'être dit, on fait relativement souvent dans les grammaires le rapprochement entre l'apposition liée et l'apposition détachée, en leur assignant le même nom et en les traitant sous la même rubrique. Dans la grande grammaire suédoise écrite par Teleman et al. (I999), par exemple, l'apposition détachée est décrite comme non restrictive et l'apposition liée comme normalement restrictive (I999, tome 3 : I IO). 
Or, la question de savoir quand on utilise l'une ou l'autre construction n'est pas beaucoup discutée, ni par Teleman et al. (I999), ni ailleurs. Parmi les comparaisons explicites que nous avons trouvées, on peut mentionner Halmøy (200I : 249), qui, à propos le type de construction où le Npr est détaché, soutient que " ce qui importe, c'est la fonction sociale du personnage ». La variante où le Nc est détaché représente selon elle « la configuration canonique du français ». Celle-ci serait « de loin la plus fréquente lorsqu'il s'agit de la première mention des personnages ». Dans Noailly (I990: I43ss), il est proposé que la différence entre les deux consiste en ce que l'apposition à Nc détaché présente une information nouvelle, alors que dans l'apposition liée, la relation entre les deux $\mathrm{N}$ n'est que rappelée.

Ces propos impliquent en quelque sorte que le journaliste ferait toujours un choix conscient lié à son intention communicationnelle, prenant en compte ses connaissances préalables, ainsi que peut-être les connaissances du lecteur. Cette conception peut vraisemblablement expliquer le choix d'apposition dans certains cas, mais nous pensons que, le plus souvent, le choix entre les trois types se fait sans réflexions communicationnelles. Si on se donne comme point de départ les points de vue d'I. Korzen (2006) et d'Acuña-Fariña (2009), présentés dans l'introduction, selon lesquels le $\mathrm{Nc}$ et le $\mathrm{Npr}$ dans l'apposition liée forment une unité indivisible mais où le lien entre les deux peut rompre si le locuteur ressent que le Nc est référentiellement saturés, on obtient une hypothèse qui explique comment le choix entre les trois constructions se fait. L'exemple suivant illustre cette hypothèse :

(5) Le prix Nobel de médecine 20 I I a été décerné à l'Américain Bruce Beutler, au Français d'origine luxembourgeoise, Jules Hoffmann et au Canadien Ralph Steinman, a annoncé lundi le comité Nobel. (L'Express, O3/IO/2OII)

Le journaliste présente dans ce texte les lauréats du prix Nobel de médicine de 20I I en donnant leurs noms en combinaison avec des substantifs de nationalité. Il/elle a choisi deux appositions liées (l'Américain BB et le Canadien $R S$ ), et une apposition que nous avons qualifiée comme détachée (malgré la ponctuation non-complète : il manque une virgule après le Npr Jules Hoffman). Dans les cas où le Nc ne contient pas d'expansion, le journaliste a donc choisi l'apposition liée, alors que dans le cas où le Nc contient une expansion, postposée à la tête du Nc, il/elle a choisi une apposition détachée. Il n'y a pas de raisons de penser que 
le journaliste aurait une intention communicationnelle particulière en choisissant des constructions divergentes, ce qui montre que les descriptions de Halmøy et de Noailly ne sont pas valables (dans ce cas). Il n'est pas non plus possible d'analyser le Nc comme lié à un référent unique, ou de regarder l'apposition comme ayant une interprétation non restrictive. Encore que l'exemple fasse voir ce qu'on pourrait qualifier comme une ponctuation non conforme à la norme, il est intéressant parce que reflétant vraisemblablement l'intuition du scripteur : là où la tête du Nc et le Npr sont séparés par l'expansion, on ressent un «besoin » de segmenter la construction. La cohésion entre la tête du Nc et le Npr est, à cause de l'expansion, ressentie comme moins forte. La ponctuation non complète donne aussi une indication sur son interprétation : la virgule sépare le Nc du Npr. Ce n'est pas le Npr qui est séparé du reste de la phrase.

Notre hypothèse est dès lors que c'est la proximité physique entre la tête du Nc et le Npr qui rend l'apposition liée plus ou moins acceptable. Si le Nc contient une expansion postposée à sa tête, le scripteur interprète plus facilement le Nc comme susceptible de référer sans le Npr, et il/elle choisit une apposition détachée au lieu de l'apposition liée. L'importance de la forme de l'expansion sera reflétée dans une différence de fréquence entre les deux constructions en français et en suédois, due au fait que normalement, l'expansion du GN français se place après la tête, alors qu'en suédois, elle peut être positionnée avant ou après la tête selon sa forme. Nous examinerons, dans la section analytique, les deux facteurs discutés dans cette section : l'interprétation du Nc comme ayant un référent unique et la position/la longueur de l'expansion, et la connexion entre ces deux facteurs avec le choix d'apposition.

Ajoutons finalement qu'il existe aussi des cas où le choix de construction est effectivement un résultat de la volonté du journaliste de présenter une personne d'une certaine manière. Le référent peut par exemple être présenté comme déjà connu par le lecteur, ce qui sera discuté en connexion avec les exemples (I4) et (2I). Or, la majorité des appositions dans la langue journalistique est mieux décrite comme neutre de ce point de vue : il s'agit simplement d'une combinaison d'un $\mathrm{Npr}$ et d'un Nc dont l'ordre des segments et l'absence ou la présence du détachement ne reflètent aucune intention communicationnelle particulière, ou plutôt une structuration informationnelle neutre. La discussion des appositions qu'il convient d'étudier dans une perspective communicationnelle sera pour une autre étude. 


\section{Méthode, délimitations et corpus}

\subsection{Délimitations}

\subsubsection{La détermination du Nc}

La concurrence entre les trois types d'appositions constitue le sujet de l'article. Cette concurrence est en principe possible lorsque le Nc prend une forme définie. Seront dès lors incluses dans l'étude les constructions à $\mathrm{Nc}$ à déterminant défini et possessif, et exclues les constructions à Nc à déterminant indéfini. En suédois, la détermination définie apparaît en deux formes (Dahl, 2004 : I 47) : l'une agglutinée au N tête (par ex. «tv-stjärnan »), et l'autre prenant la même forme que le démonstratif den, employée en combinaison avec une épithète ("den amerikanska tv-stiärnan ». De plus, il existe en suédois une forme définie où le Nc est déterminé à son tour par un GN au génitif, illustrée ici :

(6) Svenskt Näringslivs chefsekonom Stefan Fölster Svenskt Näringslivs chefsekonom, Stefan Fölster Stefan Fölster, Svenskt Näringslivs chefsekonom

Le Nc à déterminant démonstratif constitue certes une forme définie mais ne semble presque pas apparaître dans l'apposition liée, et ne sera pas étudié. Les cas à Nc à déterminant zéro ont une fréquence très élevée dans la prose journalistique, et peuvent en effet être regardés comme des concurrentes importantes des appositions liées à déterminant défini. Ils sont inclus dans l'étude malgré le fait qu'ils ne démontrent pas la possibilité théorique de concurrence :

(7) Français: Madame Berger / ? chef Berger

* chef des études économiques d'Euler Hermes, Karine Berger

Karine Berger, chef des études économiques d'Euler Hermes

Suédois : ordförande Lina Ullerstam (ou bien ordföranden Lina Ullerstam) / ? chef Lina Ullerstam

*ordförande för föreningen Barnlängtan, Lina Ullerstam

Lina Ullerstam, ordförande för föreningen Barnlängtan

L'exemple montre que l'apposition liée à déterminant zéro est possible dans certains cas : en français, en principe en connexion avec monsieur, madame, mademoiselle, et en suédois en connexion avec certains $\mathrm{Nc}$ qualifiés comme des titres. Certains items existent en deux variantes (par ex. ordförandelordföranden), d'autres dans la version définie (kronprinsessan), et encore d'autres dans la version zéro (kung). (Voir Teleman et al. I999, tome 3 : I I9.) Mais ces propos n'ont pas d'importance dans 
notre cas. Concernant le déterminant zéro, c'est l'apposition où le $\mathrm{Nc}$ est détaché qui constitue le groupe important numériquement et qui nous intéresse dans ce travail.

\subsubsection{L'expansion du Nc}

Dans nos matériaux, l'apposition de loin la plus fréquente dans les deux langues est l'apposition liée à déterminant défini dont le Nc manque d'expansion. Or, comme nous nous intéressons en premier lieu à l'importance de la forme de l'expansion pour le choix de construction, ces occurrences ne seront pas étudiées : les cas où le Nc ne contient pas d'expansion ne sont pas inclus dans l'étude. A propos de ces occurrences, on peut dire qu'une construction à détachement n'est que rarement le choix ${ }^{6,7}$. L'apposition liée est le choix normal. La possibilité d'employer une forme détachée est liée à l'interprétation du Nc comme susceptible de constituer un élément référentiel. Dans le cas où le Nc manque d'expansion, cette possibilité est le plus souvent limitée. La différence est montrée par (8) et (9):

(8) Le producteur Val Lewton, spécialiste des films d'horreur, lui propose de terminer La Malédiction des hommes-chats... (Le Monde, I 5/09/2005)

(9) ... ce $23 \mathrm{e}$ James Bond [...] a bien failli ne jamais voir le jour. [...] Après refinancement, les producteurs, Barbara Broccoli et Michael Wilson, ont pu reprendre leurs travaux (Le Figaro, O3/I I/20I I)

C'est donc le type d'apposition montré dans (8) qui est largement majoritaire. Dans ce cas, le Nc le producteur doit être lié au Npr pour former une expression référentielle. Le type dans (9) est peu fréquent. Dans ce contexte, les producteurs s'interprète comme les producteurs $d u 23^{\mathrm{e}}$ James Bond (cp. les référents "inférables » ( inferrable») de Prince, I98I), ce qui constitue une expression susceptible d'être interprétée comme référentielle. Le Nc peut dès lors être détaché du Npr. Dans le cas où le Nc est pourvue d'expansion, il est plus probable qu'il s'interprète comme une expression référentielle, et ce sont ces cas qui seront étudiés ici ${ }^{8}$.

\subsection{Corpus}

En ce qui concerne le corpus, nous avons extrait des appositions d'un certain nombre d'articles ( 300 articles français et 316 articles suédois) tirés des versions en ligne de six journaux français et six journaux suédois (L'Express, Le Figaro, Libération, Le Monde, Nice-Matin, Paris Match et Aftonbladet, Dagens Nyheter, Fokus, OmVärlden, Svenska 
Dagbladet, Sydsvenskan). Les articles ont été choisis plus ou moins au hasard, mais dans le but d'avoir des sujets différents et des journalistes différents représentés dans le corpus. De ces articles, 900 appositions françaises et 900 appositions suédoises ont été extraites, des appositions avec expansion.

Les appositions ont été divisées en les trois groupes illustrés dans (2) $-(4)$.

\section{Analyse}

Le Tableau I montre comment se répartissent les 900 appositions sur les trois groupes :

Tableau 1. La répartition des appositions à expansion sur les trois groupes

\begin{tabular}{lrrrr}
\hline & NcNpr & Npr[dét]Nc & Nc[dét]Npr & $\Sigma$ \\
\hline Français & 240 & 449 & 2 I I & 900 \\
Suédois & 522 & 277 & IOI & 900 \\
\hline
\end{tabular}

On constate une nette préférence pour les formes à détachement en français par rapport au suédois, où l'apposition liée domine. On observe également qu'environ la moitié des appositions françaises sont du type où le Nc est détaché. En suédois, l'apposition à Npr détaché est peu fréquente.

Nous discuterons ci-dessous l'interprétation référentielle du Nc, et ensuite la longueur et la position de l'expansion.

\subsection{L'interprétation sémantico-pragmatique du Nc}

Soulignons, avec Kleiber (I98 I : I3 I), qu'une « expression linguistique, étant constituée d'items lexicaux, ne peut, quelle qu'elle soit, identifier un particulier sans l'aide de connaissances extra-linguistiques ». Le Nc ne peut donc pas à lui seul viser un référent unique. Or, certains types de Nc sont plus aptes que d'autres à être interprétés comme référant à une entité unique, tandis que d'autres sont typiquement interprétés comme susceptibles d'avoir plusieurs référents. Les premiers contiennent des têtes comme par exemple président, chef, premier ministre, etc. Les deuxièmes ont, selon notre expérience, souvent, en français, la forme dét. + $N+$ adj. (en suédois dét. + adj. + N) (par ex. le poète syrien / den syriske poeten) où ni le $\mathrm{N}$ ni l'adjectif ne sont typiquement associés à un référent 
unique. Dans un grand nombre de cas, on ne peut pas savoir si un $\mathrm{Nc}$ a plusieurs référents ou non. Une expression comme le correspondant royal du journal [News of the World] 9 ne donne pas d'indications quant à son éventuelle référence unique. Vraisemblablement, ni le journaliste ni le lecteur moyen de Nice-Matin disposent de connaissances approfondies à propos du nombre de correspondants royal de News of the World en 2007 . On peut à partir de ces propos déduire qu'il n'est pas possible de faire un lien univoque entre l'emploi d'un certain type d'apposition et l'unicité du référent du Nc, tout simplement parce que ce dernier s'interprète en contexte. On verra aussi que le choix de construction même influence l'interprétation référentielle. Ceci dit, on examinera maintenant des exemples des trois types d'appositions.

Dans les deux langues, l'apposition liée semble être employée indépendamment de l'interprétation du Nc comme ayant potentiellement un référent unique ou non :

(10) Français : son dernier album Goodbye Lullaby (Figaro, 26/07/20I I) le porte-parole du gouvernement Ilias Mossialos (Libération, 03/IO/20I I) le couple d'acteurs américains Tom Cruise et Katie Holmes (Libération, 29/06/20I2)

Suédois : hennes nye man Daniel Heckscher (Aftonbladet, 02/03/20I2) OmVärldens bildredaktör Julia Björne (OmVärlden, I 5/03/20I I) det irländska stjärnskottet Saoirse Ronan (Dagens Nyheter, o8/o7/20 I I)

On voit que l'apposition liée est utilisée dans les cas où le Nc s'associe à un référent unique, comme dans son dernier album et hennes nye man, dans les cas où la situation interprétative est liée aux éventuelles connaissances du journaliste / du lecteur (le porte-parole du gouvernement et OmVärldens bildredaktör), aussi bien que dans les cas où le Nc peut viser plusieurs référents (le couple d'acteurs américains et det irländska stjärnskottet). Cette souplesse d'emploi est digne d'attention puisqu'elle explique, en partie, pourquoi l'apposition liée est tellement fréquente : elle s'emploie indépendamment de l'interprétation référentielle du Nc.

On tourne maintenant l'attention vers l'apposition à Npr détaché :

(I I) Özil hade ett väldigt gott centralt understöd av klubbkamraten från Santiago Bernabeu, Sami Khedira. (Svenska Dagbladet, 26/06/20I2)

(I2) Mellan åkerlapparna står de vackra sädmagasinen, hórreos, som förstenade tidsresenärer, byggda på pålar för att hålla mössen borta. (Sydsvenskan, I $7 / 06 / 2006$ )

(I3) le président iranien, Mahmoud Ahmadinejad (L'Express, I9/06/20I2) 
En comparaison avec le groupe d'appositions liées, il est clair que ce groupe contient davantage d'exemples où le Nc peut s'associer à un seul référent. En même temps, on peut dire qu'en utilisant la construction à Npr détaché, le journaliste guide le lecteur vers une interprétation du Nc comme ayant le référent unique visé par le Npr. Dans (I I), il existe potentiellement plus d'un coéquipier venant du club en question, mais on tend à regarder le Nc comme se référant justement à ce coéquipier à qui réfère le Npr, donc comme ayant un référent unique. Dans (I2), le lecteur doit interpréter le Nc en fonction de son contexte. Il y a dans le monde d'autres magasins à blé pouvant répondre à la description du Nc, mais dans ce cas, le Nc s'interprète comme se référant au type de magasin qui existe dans la région discutée dans le texte. Pourtant, en suédois, la construction n'apparaît pas fréquemment en connexion avec des Nc à référence unique "inhérente » comme ceux dont la tête est par ex. president, chef, generalsekreterare, etc. C'est là un point de divergence par rapport au français, où cet emploi de la construction est répandu (I3).

Quant à la question de savoir si on préfère écrire, en français, le président iranien, Mahmoud Abmadinejad, avec virgule, ou le président iranien Mahmoud Abmadinejad, sans virgule, il est difficile d'y répondre. Au vue des raisonnements menés ci-dessus, on comprend que la difficulté d'établir l'unicité du référent rend plus ou moins impossible de faire des statistiques là-dessus. Nous nous contentons de conclure que les deux types sont fréquents en français, et le journaliste peut vraisemblablement choisir l'un des deux selon son goût personnel. En suédois, la première variante est largement préférée.

$\mathrm{La}$ troisième apposition est celle où le $\mathrm{Nc}$ est détaché. Pour cette variante, il convient de rappeler que le Nc peut soit prendre une forme définie soit le déterminant zéro ${ }^{\text {Io }}$. Dans le deuxième cas, l'absence de déterminant amène l'impossibilité du Nc de référer. Des exemples où le Nc est susceptible de référer sont donnés dans (I4) :

(I4) Français : L'explosif découvert à Leeds, le TATP, est fréquemment employé par les islamistes. Il est du même type que celui dont Richard Reid, "l'homme aux chaussures piégées " était en possession. ${ }^{\text {II }}$ ( Le Monde, , 6/07/2005)

Suédois : Det finns många åsikter om Assange, den fyrtioårige australiske programmeraren och internetaktivisten som blev världsberömd över en natt i egenskap av grundare och talesman för Wikileaks. (Dagens Nyheter, 29/02/2012)

Il semble peu problématique d'interpréter ces Nc comme ayant un seul référent. De l'autre côté, cette interprétation est vraisemblablement 
inhérente à la construction même : on interprète nécessairement le $\mathrm{Nc}$ comme ayant le même référent unique que le Npr. Cela est montré par la réécriture de ( 5 a) :

( 5 a) Le couple d'acteurs américains Tom Cruise et Katie Holmes a entamé une procédure de divorce, après un peu plus de cinq ans de mariage, a-t-on appris auprès de leurs représentants. (Libération, 29/06/20I2)

(I5b) Tom Cruise et Katie Holmes, le couple d'acteurs américains, a entamé...

La version dans ( ${ }_{5}$ b) est acceptable, malgré le fait que le Nc le couple d'acteurs américains pourrait référer à d'autres couples. Le référent unique (ou dans ce cas les deux référents) est déjà présenté dans le contexte de gauche, par le(s) $\mathrm{Npr}(\mathrm{s})$, et ainsi l'unicité du référent du Nc est assurée. Ce qui différencie les deux versions, c'est que dans ( I 5 b), le scripteur présente les référents des Npr comme connus de la part du lecteur, ce qui n'est pas le cas dans (I 5 a).

Tirons la conclusion que c'est, le plus souvent, dans les deux langues, l'apposition liée qui fait voir un Nc référentiel qui s'interprète comme non unique, par rapport aux autres types d'appositions, dont le Nc s'interprète plus souvent comme ayant un seul référent. Or, les constructions mêmes aident ces interprétations.

\subsection{L'importance de la forme de l'expansion du Nc}

Dans cette section, nous discuterons l'importance de la forme de l'expansion pour le choix de construction. Pour l'anglais, Acuña-Fariña (2009 : 472) soutient que lorsque le $\mathrm{Nc}$ "starts sounding like a referential NP ", il devient nécessaire de dissocier le Nc du Npr. Notre variante de cette proposition est que lorsque la tête du Nc et le Npr se trouvent séparés par une expansion, le Nc est souvent interprété comme un GN référentiel, et il est dès lors plus probable que le scripteur choisit une structure à détachement. En suédois, l'expansion du GN peut se placer avant ou après la tête, selon la forme de l'expansion (les syntagmes prépositionnels, SP, et les subordonnées se placent après la tête). On peut donc comparer les cas à expansion antéposée à ceux à expansion postposée, pour examiner le lien de ce facteur avec le choix d'apposition. En français, l'expansion du GN est presque toujours ${ }^{\mathrm{I2}}$ positionnée après la tête, ce qui implique que c'est plutôt la longueur de l'expansion qui a de l'importance dans cette langue.

Dans la partie suédoise du corpus, il y a 522 occurrences d'appositions liées, dont 7 occurrences qui font voir une expansion postposée 
(voir l'ex. (I6), et 277 occurrences d'appositions à Nc détaché, dont 229 qui font voir une expansion postposée (l'ex. (I 7)). Quand l'expansion est postposée à la tête du Nc, on évite donc l'apposition liée. C'est dans ce cas l'apposition à Nc détaché qui constitue le choix préférér ${ }^{13}$.

(I6) världens största tillverkare av luftkonditioneringsapparater Dong Mingzhu (OmVärlden, 29/or/20I2)

(I7) Jon Åström, chef för den konsulära enheten på utrikesdepartementet (Aftonbladet, 30/06/20I2)

Pour le français, le facteur "position » de l'expansion n'est donc pas, dans la grande majorité des cas, susceptible d'influencer le choix d'apposition. Si on regarde par contre la longueur du Nc, on peut constater que l'expansion de l'apposition liée est assez souvent composée d'un seul mot, par exemple un adjectif de nationalité (I8), ou d'une suite de mots qui peut correspondre à un mot composé en suédois (I9). Or, il existe aussi en français un grand nombre de cas où il y a une expansion relativement longue antéposée au mot tête du Nc (20).

(I8) la journaliste américaine Diane Sawyer (Paris Match, 28/og/20II)

(19) le groupe de Philadelphie The Roots (Le Monde, o6/o7/20II)

(20) le site internet spécialiste des bonnes affaires Groupon (Le Monde, 05/II/2OII)

Il est cependant facile de constater que le choix préféré dans le cas où l'apposition contient une expansion d'une certaine longueur est le type dont le Nc est détaché :

(2I) Benoît Magimel, moniteur d'auto-école fracassé par la disparition de sa femme (Figaro, 24/09/20II)

Ces observations mènent à la conclusion qu'en suédois, on tend à éviter l'apposition liée dans le cas où celle-ci ferait voir une expansion intercalée entre la tête du Nc et du Npr (mais cette construction n'est pas totalement absente dans les matériaux), alors qu'en français, le scripteur a plus de liberté dans le choix de construction : on peut avoir dans l'apposition liée une expansion relativement longue, mais, globalement, dans le cas où l'expansion contient un grand nombre de mots, c'est l'apposition à Nc détaché qui constitue le choix le plus fréquent.

\section{Conclusion}

Pour conclure, disons quelques mots sur la question de savoir s'il y a des situations où les trois constructions se substituent l'une à l'autre, 
sans changement de sens. On peut répondre par l'affirmative. Il y a certaines situations où l'auteur peut choisir l'une des constructions selon son goût personnel, sans que ce choix emmène nécessairement des changements dans le message. Le dernier exemple illustre l'emploi typique des appositions dans les deux langues :

(22) Suédois : Slowfoodrörelsens grundare Carlo Petrini / Carlo Petrini, grundare av Slowfoodrörelsen

$\rightarrow$ Français : le fondateur du mouvement Slow Food Carlo Petrini

$\rightarrow$ le fondateur du mouvement Slow Food, Carlo Petrini

$\rightarrow$ Carlo Petrini, (le) fondateur du mouvement Slow Food

Ceci dit, on doit souligner que la majorité des constructions appositives peut se décrire selon le principe que le choix de construction se fait selon la forme de l'expansion. Ce principe résulte dans des différences entre des cas contenant une expansion longue et ceux contenant une expansion courte ou aucune expansion du tout, mais aussi dans des différences entre le français et le suédois, où cette dernière langue utilise davantage d'appositions liées, ce qui peut s'expliquer par la position normale de l'expansion avant sa tête. L'unicité du référent du Nc a aussi un rôle à jouer : on tend à éviter l'apposition détachée dans le cas où le Nc s'interprète typiquement comme ayant plusieurs référents.

Nous avons mentionné les discussions concernant la question de savoir quel mot est la tête de l'apposition liée. D'un point de vue grammatical, la présente étude n'a rien à apporter à ces discussions. Or, d'un point de vue pragmatique/communicationnelle, il semble clair que c'est, dans un grand nombre des exemples étudiés, le Npr qui assure le lien avec le référent. Ceci vaut pour l'apposition liée, mais également pour l'apposition à $\mathrm{Nc}$ détachée et, à notre avis, aussi pour certaines appositions à Npr détaché. Dans ce sens, le Npr constitue le centre des constructions, et on peut effectivement décrire le Nc comme "adjective-like ", comme le fait Aarts (2007 : I29ss). Dans le cas le président Obama, on identifie donc Obama comme le président, plutôt que d'identifier le président dont le nom est Obama.

\section{Notes}

I. Il existe bien évidemment d'autres tours présentatifs que les appositions, par ex. « Nelly Martin, de la Marche Mondiale des femmes, ... » (Le Figaro, 29/09/20I I), "Frédéric Stella est un désobéissant civil du Loiret. Ce consultant en gestion de l'information de 53 ans..." (Libération, 07/07/20II), "Laurence, qui représente la troisième génération des Addeo, connaît tous les commerçants italiens du quartier... " (Le Figaro, 09/o9/20II). 
2. Acuña-Fariña (2009 : 458) écrit: " canonical loose appositions ought to be seen as pragmatic 'insertions' which do not belong to the syntactic structure of a sentence ».

3. Rioul ( I983) parle d'apposition "intégrée "; dans Teleman et al. (I999), on utilise les termes "fast» et "lös apposition », et pour l'anglais, Acuña-Fariña parle de «close » et "loose apposition».

4. Dans Riegel et al. (I994 : I86) la construction est brièvement discutée. Aucun terme spécifique n'y est donné, mais on parle de "noms épithètes ", et c'est le Npr qui est qualifié d' "épithète ». Un autre terme relativement commun est celui de "juxtaposition ", utilisé entre autres dans Togeby (I982).

5. Acuña-Fariña (2009 : 47I) parle d'un « constructional 'freezing point' that depends on the referential saturation of the first unit " (le terme "saturation" est emprunté à Grimshaw, I990: I7ss.). Quelques exemples que donne AcuñaFariña (2009 : 47I-472) : " the unique poet Burns ", "the Jaguar boss Tom Walkenshaw ", "the stubborn Jaguar boss Tom Walkenshaw ", "the increasingly desperate Jaguar boss Tom Walkenshaw ". Les deux premiers seraient acceptables, mais pas les deuxièmes. D'après nous, on peut émettre des réserves quant à l'intuition d'Acuña-Fariña concernant la non-acceptabilité des deux derniers exemples.

6. Pour avoir une vue de leur fréquence : les appositions liées sans expansion comprennent dans notre corpus environ entre 900 et Ioo० occurrences dans chaque langue. Les appositions détachées sans expansion comprennent I 26 occurrences et 26 occurrences en français et en suédois respectivement, ce qui est une différence notable.

Or, il convient de signaler quelques observations à propos de ces appositions. Premièrement, ce groupe contient un grand nombre d'occurrences comme les années I970, le rang 3, la chambre 606, etc. Se pose alors la question de savoir si celles-ci sont à regarder comme le même type d'apposition que par ex. le président Obama. Deuxièmement, dans certaines expressions géographiques, comme l'avenue Victor-Hugo, on se demande s'il est question d'une avenue appelée «Victor-Hugo » ou d'une avenue appelée « avenue Victor-Hugo ». Parfois, l'emploi de majuscules peut probablement être regardé comme un indice pour interpréter "le $\mathrm{Nc}$ » comme faisant partie du nom propre : la Galerie Yvon Lambert serait dès lors une galerie portant le nom «Galerie Yvon Lambert ».

Il y a donc, pour ce groupe d'appositions, des questions à discuter, et c'est là aussi une des raisons pour laquelle ces cas seront ici laissés de côté.

7. En connexion avec le déterminant possessif, les variantes à détachement sont relativement plus fréquentes, parce qu'elles sont susceptibles de s'interpréter comme des expressions référentielles sans le Npr. 
8. Dans (9), c'est la construction qui indique qu'on doit chercher dans le contexte pour comprendre la référence de les producteurs. Certains autres items lexicaux indiquent sémantiquement que la référence peut se comprendre à travers le contexte, comme dans le cas de le propriétaire / ägaren. On est nécessairement le propriétaire de quelque chose déjà mentionné dans le contexte. Cela vaut aussi pour des relations familiales.

9. Le Nc le correspondant royal du journal en contexte: « [...] les peines de prison prononcées en 2007 contre le correspondant royal du journal Clive Goodman, et le détective privé Glenn Mulcaire, impliqués dans un premier scandale, 'n'avaient pas mis fin aux problèmes'. » (Nice-Matin, o7/o7/20I I)

Io. En français, I 43 des 449 occurrences prennent une forme définie, en suédois, 87 des 277 occurrences.

I I. C'est là encore un exemple de ponctuation « incomplète ».

I2. Des adjectifs antéposés à la tête du Nc dans nos matériaux sont ancien, dernier, célèbre, nouveau, mythique, etc.

I3. Dans cette discussion, nous avons exclu les appositions à Npr détaché, par manque d'espace, mais aussi, premièrement, parce qu'en suédois, ceux-ci constituent un groupe peu important numériquement, et, deuxièmement, font souvent voir des Nc « doubles ", par exemple : «sin huvudmotståndare, talmannen John Boehner" (Fokus, I7/06/20II). Dans ce cas, il semble plausible que le détachement sépare les deux Nc, plutôt que le premier Nc et le Npr.

\section{Références}

\section{Corpus}

Aftonbladet (www.aftonbladet.se)

Dagens Nyheter (www.dn.se)

Fokus (www.fokus.se)

Le Figaro (www.lefigaro.fr)

L'Express (www.lexpress.fr)

Le Monde (www.lemonde.fr)

Libération (www.liberation.fr)

Nice Matin (www.nicematin.com)

OmVärlden (www.sida.se/omvarlden)

Paris Match (www.parismatch.com)

Svenska Dagbladet (www.svd.se)

Sydsvenskan (www.sydsvenskan.se) 


\section{Ouvrages cités}

Aarts, Bas. 2007. Syntactic gradience : The nature of grammatical indeterminacy. Oxford : Oxford University Press.

Acuña-Fariña, Juan Carlos. 2009. "Aspects of the grammar of close apposition and the structure of the noun phrase ". English Language and Linguistics, I 3 : 3. Cambridge : Cambridge University Press. 453-48 I.

Dahl, Östen. 2004. "Definite articles in Scandinavian: Competing grammaticalization processes in standard and non-standard varieties ». In: Bernd Kortmann (dir.). Dialectology meets Typology : Dialect grammar from a cross-linguistic perspective. Trends in Linguistics. Studies and monographs. Berlin : Mouton de Gruyter. I47-I 80.

Eriksson, Olof. 1993. La phrase française. Essai d'un inventaire de ses constituants syntaxiques. Göteborg : Acta Universitatis Gothoburgensis, Romanica Gothoburgensia XLII.

Forsgren, Mats. I99I. «Éléments pour une typologie de l'apposition en linguistique française ". Actes du XVIII Congrès International de Linguistique et de Philologie Romanes, Université de Trèves I986, II, Linguistique théorique et linguistique synchronique. Tübingen : Max Niemeyer Verlag. 597-6I2.

- 2000. "Apposition, attribut, épithète : même combat prédicatif ?". Langue française $\mathrm{I} 25 \cdot 30-45$.

— 200I. "Le référent existe - je veux bien, mais comment ? ". Langage et référence. Mélanges offerts à Kerstin Jonasson à l'occasion de ses soixante ans. In : Kronning, Hans et al. (dir.). Uppsala : Acta Universitatis Upsaliensis, Studia Romanica Upsaliensia. I73-I85.

Grimshaw, Jane. I990. Argument structure. Cambridge, MA : MIT Press.

Halmøy, Odile. 200I. "Les satellites des 'Noms propres de l'actualité' dans la presse française et norvégienne ". Langage et référence. Mélanges offerts à Kerstin Jonasson à l'occasion de ses soixante ans. In : Kronning, Hans et al. (dir.). Uppsala : Acta Universitatis Upsaliensis, Studia Romanica Upsaliensia. 245-253.

Hannay, Mike. \& Evelien Keizer. 2005. " A Discourse Treatment of English Non-Resrictive Nominal Appositions in Functional Discourse Grammar ». In: Lachlan Mackenzie, J. \& María de los Angeles GómezGonzález (dir.). Studies in Functional Discourse Grammar. Bern : Peter Lang. I 59-I 94 .

Hockett, Charles F. I95 5. «Attribution and apposition in English ». American Speech, 30. 99-102. 
Jonasson, Kerstin. I994. Le nom propre. Constructions et interprétations. Louvain-la-Neuve : Duculot.

Keizer, Evelien. 2005. "The discourse function of close appositions ". Neophilologus, 89. 447-467.

Kleiber, Georges. 1981. Problèmes de référence : descriptions définies et noms propres. Paris : Klincksieck.

Korzen, Hanne. 2006. "Appositions and related constructions in French and Danish ». In : Peter Colliander (dir.). Appositions in Selected European Languages. Copenhagen Studies in Language, 33, Samfundslitteratur Press. $53^{-92 .}$

Korzen, Iørn. 2006. "Appositions (and similar constructions) in Italian ». In : Peter Colliander (dir.). Appositions in Selected European Languages. Copenhagen Studies in Language, 33. Samfundslitteratur Press. 93-I40.

Meyer, Charles F. I992. Apposition in contemporary English. Cambridge : Cambridge University Press.

Noailly, Michèle. 1990. Le substantif épithète. Paris : Presses Universitaires de France.

Prince, Ellen, F. I98 I. « Toward a Taxonomy of Given-New Information ». In : Peter Cole (éd.). Radical Pragmatics. New York/London : Academic Press. 223-255.

Riegel, Martin, Jean-Christophe Pellat \& René Rioul. I994. Grammaire méthodique du français. Paris : Presses Universitaires de France.

Rioul, René. 1983. "Les appositions dans la grammaire française ". L'information grammaticale, I8. 2 I-29.

Teleman, Ulf, Staffan Hellberg \& Erik Andersson. I999. Svenska Akademiens grammatik. Stockholm : Norstedts Ordbok.

Togeby, Knud. I982. Grammarie française Volume I: Le Nom. Etudes Romanes de l'Université de Copenhague. Copenhague : Akademisk Forlag. 